\title{
Anormalidades sensoriais: Olfato e paladar
}

\author{
Sensorial abnormalities: Smell and taste
}

\section{Francisco Xavier Palheta Neto', Mauricio Neres Targino², Victor Soares Peixoto², Flávia Barata Alcântara², Camila Corrêa de Jesus ${ }^{3}$, Dalila Costa de Araújo30 ${ }^{3}$ Eduardo Flávio de Lacerda Marçal Filho3.}

1) Mestrado em Otorrinolaringologia. Doutor em Neurociências. Preceptor da Residência Médica em Otorrinolaringologia do Hospital Universitário Betina Ferro de Souza da Universidade Federal do Pará. Professor Adjunto da Universidade do Estado do Pará e da Universidade Federal do Pará

2) Aluno do Quinto Ano do Curso de Medicina. Universidade do Estado do Pará

3) Aluna do Quarto Ano do Curso de Medicina. Universidade do Estado do Pará

Instituição: Centro de Otorrinolaringologia do Pará - COP Belém / PA - Brasil

Endereço para correspondência: Francisco Xavier Palheta Neto - Centro de Otorrinolaringologia do Pará - Avenida Conselheiro Furtado, 2391 , salas 1508 e 1608 - Edifício Belém Metropolitan-Bairro: Cremação - Belém/PA - Brasil-CEP 66040-100 - Telefone: (+5591) 3249-9977/3249-7161/9116-0508-E-mail: franciscopalheta@ hotmail.com Artigo recebido em 26 de Outubro de 2009. Artigo aprovado em 17 de Novembro de 2009

\section{RESUMO}

Introdução: Anormalidades do paladar e do olfato comprovaram ser um tema bem mais complexo do que se reconhecia anteriormente. Diversas entidades nosológicas cursam com alterações olfatórias e gustatórias, podendo ser congênitas ou adquiridas.

Objetivo: Analisar os principais aspectos das disfunções olfatórias e gustatórias.

Método: Foram utilizadas as bases de dados informatizados para a coleta de dados, tendo como palavras-chave "alteração", "olfato" e "paladar". Realizou-se também busca nãosistemática em publicações científicas e livros médicos.

Revisão da Literatura: Disfunções olfatórias e gustatórias possuem etiologia variada, destacando-se as doenças nasais e sinusais obstrutivas, infecções do trato respiratório superior, traumatismo cranioencefálico, envelhecimento, exposição a tóxicos e algumas medicações, neoplasias nasais ou intracranianas, patologias psiquiátricas e neurológicas, iatrogenia, causas idiopáticas e congênitas. Anamnese detalhada, exame físico atencioso e exames complementares adequados são importantes para o diagnóstico dessas alterações.

Conclusão: Disfunções olfatórias e gustatórias frequentemente ocorrem juntas. A detecção precoce de tais disfunções pode levar a um tratamento mais efetivo, retardando a progressão das doenças que as ocasionam e atenuando a severidade dos sintomas. Em muitos casos o tratamento dessas alterações não é fácil e é necessária uma cooperação interdisciplinar entre o otorrinolaringologista, endocrinologista, neurologista, psiquiatra entre outros.

Palavras-chave: olfato, transtornos do olfato, paladar, distúrbios do paladar, modalidades sensoriais, otolaringologia.

\section{SUMMARY}

Introduction: Taste and smell abnormalities have proven to be an extremely more complex subject than previously regarded. Wide-ranging nosologic entities arise along with smell and taste alterations, and they can be congenital or acquired. Objective: Analyze the main features of smell and taste dysfunctions.

Method: Automated databases were used to collect data, by searching keywords like 'alteration', 'smell', and 'taste'. A nonsystematic search was also made in scientific printings and medical books.

Literature Review: Smell and taste dysfunctions have a vast etiology, the most significant of which are obstructive nasal and sinusal disease, infections of the upper respiratory tract, cranioencephalic trauma, aging, exposure to toxics and some drugs, nasal or intracranial neoplasias, psychiatric and neurological pathologies, iatrogenic disease, idiopathic and congenital causes. A detailed anamnesis, a careful physical examination and supplementary evaluations are important for the diagnosis of these alterations.

Conclusion: As a rule, smell and taste dysfunctions occur in a combined way. The early discovery of such dysfunctions can lead to a more efficient treatment, making the progress of diseases causing them retard and the symptoms less severe. In many cases, treating these alterations is not easy and there needs to be a multidisciplinary cooperation among the otorhinolaryngologist, endocrinologist, neurologist, psychiatrist, among others.

Keywords: smell, smell disorders, taste, taste disorders, sensory modalities, otorhinolaryngology. 


\section{INTRODUÇÃO}

O olfato e o paladar são sentidos químicos. Os sistemas neurais que intermedeiam estas sensações, os sistemas gustatório e olfatório, estão entre aqueles filogeneticamente mais antigos do encéfalo e ao perceberem substâncias químicas na cavidade oral e nasal trabalham conjuntamente $(1,2)$.

As sensações surgem pela interação de moléculas com os receptores da olfação e gustação. Como os impulsos se propagam para o sistema límbico (bem como para as áreas corticais superiores), certos odores e gostos podem desencadear intensas respostas emocionais ou afluxo de memórias (1).

A importância do paladar reside no fato de que ele permite a umindivíduo selecionar substâncias específicas de acordo com os seus desejos e, frequentemente, de acordo com as necessidades metabólicas dos tecidos corpóreos (3).

A olfação, mais ainda que a gustação, tem a qualidade afetiva de ser agradável ou desagradável. Por isso, a olfação é, provavelmente, mais importante do que a gustação para a seleção de alimentos (3).

Sabe-se que a gustaçãoé sobretudo uma função dos corpúsculos gustativos da boca, masé experiência comum que o sentido do olfato contribui fortemente para a percepção do gosto (2). É imprescindível ressaltar a sua relação com a gustação, pois sem o olfato não sentimos de forma adequada o sabor dos alimentos, perdendo assim o apetite e o prazer com a alimentação (4).

Os botões gustativos diminuem com a idade e as papilas gustativas, que atingem seu clímax de desenvolvimento na puberdade, começam a atrofiar na mulher entre 40-45 anos e no homem aos 50 anos (5).

Quantoà olfação, o declínio da sensibilidade olfativa com a idade pode ser resultante de degeneração de células centrais e ser independente de modificações periféricas do aparelho olfativo. Porém, a capacidade regenerativa do epitélio olfatório declina com a idade (5). A qualidade e intensidade da percepção olfatória dependem do estado anatômico e funcional do epitélio nasal e dos sistemas nervosos central e periférico. Rinites e resfriados prolongados podem causar hiposmia (perda moderada da sensibilidade olfativa) $(4,6)$.

As alterações do olfato e do paladar podem estar relacionadas com deformidades nasosseptais, polipose nasal e congestão nasal crônica decorrente de rinites alérgicas e não-alérgicas (7).
Um dano ao sistema olfatório, como resultado de um traumatismo craniano, ou mesmo, um resfriado comum, o qual impede a condução de moléculas transportadas pelo ar nas cavidades nasais, pode atenuar a percepção do sabor, ainda que as sensações básicas do gosto doce, ácido, salgado e amargo estejam preservadas (2).

O olfato pode ajudar, também, no diagnóstico precoce de algumas doenças neurodegenerativas, como a doença de Parkinson. O resultado indica que o comprometimento do olfato, e, consequentemente, do paladar, é um indicativo importante para o diagnóstico precoce da doença, em uma fase na qual os sintomas motores típicos (como tremores, rigidez e lentidão na execução dos movimentos) ainda não se manifestaram (8).

Sendo assim, torna-se importante a realização de estudos das alterações do olfato e paladar para detecção precoce, e assim um tratamento mais efetivo, no intuito de retardar a progressão das doenças e possíveis complicações que possam levar a perda olfatória e gustatória e, desta forma, atenuar a severidade dos sintomas.

O objetivo é realizar uma revisão de literatura sobre os diversos aspectos relacionados às alterações do olfato e paladar.

\section{MÉTOdO}

Foram utilizadas as bases de dados informatizados para a coleta de dados, tendo como palavras-chave "alteração", "olfato" e "paladar". Realizou-se também busca nãosistemática em publicações científicas e livros médicos.

\section{Morfofisiologia}

Os sentidos de olfação e gustação fornecem um meio de avaliar moléculas voláteis no ambiente e os componentes voláteis e não-voláteis dos alimentos. Desse modo, os humanos e outros mamíferos são capazes de discriminar uma grande variedade de odores e sabores. Apesar de a capacidade olfatória dos humanos ser limitada, se comparada com a capacidade de outros mamíferos, o homem é capaz de perceber uma grande variedade de diferentes moléculas odoríferas. A sensação de sabores resulta da combinação de informações gustativas, olfatórias e somatossensórias $(9,10)$.

\section{Olfação}

No homem, o sentido da olfaçãoé, provavelmente, o menos compreendido, pois, em grande parte, pode ser 
um fenômeno subjetivo. A superfície receptora para agentes odoríferos localiza-se na parte superior da cavidade nasal e, tipicamente, tem área de superfície de apenas 2,4 $\mathrm{cm}^{2}$. As células olfatórias são neurônios bipolares derivados do sistema nervoso central. De seu pólo apical, cada neurônio estende um único dendrito para a superfície epitelial, onde o dendrito se expande em um grande botão, do qual 5-20 ć́lios finos projetam-se na camada de muco que cobre o epitélio. De seu pólo basal, cada neurônio projeta um único axônio através da placa cribiforme óssea acima da cavidade nasal para o bulbo olfatório (10).

Há especialização dos cílios dos neurônios olfatórios na detecção de odores, pois eles têm receptores específicos para agentes odoríferos, bem como a maquinaria de transdução necessária à amplificação de sinais sensórios e à geração de potenciais de ação no axônio do neurônio. $O$ muco que banha os cílios é secretado pelas células de suporte do epitélio olfatório e pelas glândulas de Bowman, que ficam sob o epitélio e possuem dutos que se abrem em sua superfície. Acredita-se que o muco forneça o ambiente molecular e iônico apropriado para a detecção de odores (9-11).

A resposta normal do neurônio a um agente odorífero consiste na despolarização e na produção de potenciais de ação. O número de neurônios que respondem varia de acordo com a concentração do agente $(9,10)$.

A informação sensória do nariz é transmitida ao cérebro através dos bulbos olfatórios. Os nervos do olfato passam pelas perfurações da placa cribiforme e entram no bulbo olfatório, que são estruturas pareadas localizadas acima e atrás das cavidades nasais e consiste em um nó emaranhado de dendritos das células mitrais e em tufo e fibras nervosas olfatórias. Os axônios mitrais e das células em tufo deixam o bulbo pelo trato olfatório e entram em regiões especializadas do córtex, sem passar, primeiro, pelo tálamo $(9,10)$.

\section{Gustação}

Células gustativas agrupadas em botões gustativos na língua, palato, faringe, epiglote e terço superior do esôfago podem detectar alguns tipos de moléculas. $\mathrm{Na}$ língua, os botões gustativos estão localizados principalmente nas papilas, que estão no epitélio (9-11).

Três tipos morfológicos de papilas são encontrados em regiões diferentes da língua. Várias centenas de papilas fungiformes, que têm uma estrutura parecida com pino, estão localizadas nos dois terços anteriores da língua. No terço posterior, estão as grandes papilas circunvaladas, cada uma delas sendo circundada por um sulco. As papilas foliadas, situadas na borda posterior da língua, são estruturas parecidas com folhas, e cada uma delas também é circundada por um sulco. Cada papila fungiforme contém de um a cinco botões gustativos, enquanto cada papila circunvalada ou foliada contém centenas de botões gustativos $(9,11)$.

Há quatro tipos de morfologicamente distinguíveis de células que são encontradas em cada botão gustativo: células basais, células escuras, células claras e células intermediárias. Acredita-se que as células basais sejam as células germinativas, das quais as outras células são derivadas.

O botão gustativo tem uma abertura pequena na superfície do epitélio, chamada de poro gustativo. As cem ou mais células gustativas em cada botão estendem microvilosidades, onde a transdução sensória ocorre. A célula gustativa é inervada por neurônios sensórios (fibras aferentes gustativas primárias) no seu pólo basal. Além disso, células gustativas, como os neurônios, são células eletricamente excitáveis, com canais de sódio, potássio e cálcio dependentes de voltagem e capazes de gerar potenciais de ação (9).

Para fins práticos, considera-se que o sistema gustativo distingue quatro qualidades de estímulos básicos: amargo, salgado, azedo e doce. O glutamato monossódico pode representar uma quinta categoria de estímulo, chamada "umami". Cada tipo de estímulo gustativo é detectado por um mecanismo diferente. Essas interações tipicamente despolarizam a célula, diretamente ou pela ação de segundos mensageiros. O potencial receptor resultante gera potenciais de ação na célula gustativa, que, por sua vez leva a um influxo de cálcio dependente de voltagem eà liberação de neurotransmissor nas sinapses formadas com fibras sensórias. Outro mecanismo alternativo pode envolver a liberação de cálcio dos estoques intracelulares (9).

Os sabores azedo e salgado envolvem permeação, ou bloqueio, de canais iônicos por íons sódio (sabor salgado) ou íons hidrogênio (sabor azedo), enquanto sabores doce e amargo parecem ser mediados em alguns casos por receptores específicos (mas em alguns casos eles podem resultar de efeitos diretos em canais iônicos) (9).

As fibras gustatórias dos dois terços anteriores da língua trafegam, primeiro, por ramos do nervo trigêmeo e, depois, pela corda do tímpano, um ramo do nervo facial. A sensação gustação do terço posterior da língua é conduzida por fibras do nervo glossofaríngeo, enquanto outras fibras da epiglote e de outras áreas cursam por ramos do nervo vago. A partir de sua entrada no troco cerebral, todas as fibras gustatórias são afuniladas para o trato solitário e, finalmente fazem sinapse na porção rostral do núcleo do 
trato solitário. Desse ponto, os axônios passam rostralmente, por vias mal definidas, para o núcleo ventromedial do tálamo e, depois, para o córtex cerebral, na região central do giro pós-central, que se enrola para dentro da fissura lateral (10).

\section{Etiologia}

Diversas entidades nosológicas cursam com alterações olfatórias e gustativas, podendo ser congênitas ou adquiridas, sendo as mais citadas na literatura: doença nasal e sinusal obstrutiva, infecções de vias aéreas superiores, traumatismo cranioencefálico, envelhecimento, causa congênita, exposiçãoa tóxicos, algumas medicações, neoplasias nasais ou intracranianas, alterações psiquiátricas, doenças neurológicas, iatrogenia e idiopática. As anormalidades do paladar e do olfato comprovaram ser um tema bem mais complexo do que se reconhecia anteriormente e também estão presentes em situações como deficiência de vitaminas (B6, B12, A) e de zinco ou de cobre, tabagismo, gravidez, anestesia geral, traumas dentários, arrinencefalia e desvios do septo nasal (12-18).

A obstrução é a causa mais comum de distúrbio olfatório. Se a obstrução é total, o indivíduo apresenta anosmia (moléculas odoríferas não atingem o epitélio olfatório), liberando a obstrução a habilidade olfatória retorna. A porção ântero-medial da parte inferior do corneto médio funciona como reguladora do fluxo aéreo para a região olfatória. Obstrução nesta área crítica por edema da mucosa, pólipos, tumores, deformidades ósseas, cirurgias entre corneto médio e septo nasal ou trauma podem diminuir ou eliminar a habilidade olfatória. Isto pode acontecer mesmo quando a cavidade inferior parece normal. Podem ocorrer em qualquer faixa etária, com predominância em mulheres. Os pacientes geralmente referem perda progressiva e gradual da olfação, flutuante, podendo ocorrer perdas agudas com infecções agudas e exposição a alérgenos $(12,13)$.

As infecções de vias aéreas superiores também constituem uma das principais causas de perda olfatória. A maioria em indivíduos entre 40 e 60 anos de idade, dos quais 70-80\% são mulheres, geralmente por obstrução do fluxo aéreo e se resolve em um período de um a três dias. Em alguns poucos casos a olfação não retorna ao normal. À biópsia, pode haver metaplasia, com diminuição ou ausência de receptores olfatórios e com substituição por epitélio respiratório em alguns casos. A perda olfatória é proporcional à perda neuronal e o prognóstico é pobre. Um terço recupera-se espontaneamente com ou sem tratamento, ocorrendo mais frequentemente hiposmia que anosmia. Raramente ocorre fantosmia (percepção de um odor que não é real) $(16,17,19)$.
Traumatismos cranioencefálicos podem ocasionar danos aos nervos olfativos na lâmina cribiforme devido as forças de golpe ou contragolpe. Em adultos a perda da olfação é de 5-10\%, já em crianças é de 1,3-3,2\%. É mais prevalente no sexo masculino, com cerca de 60\% dos casos. Em geral o grau de perda está associadoà severidade do trauma, o que não significa dizer que um trauma mínimo não possa estar associado à anosmia. O início da perda geralmente é imediato, mas alguns pacientes só percebem após alguns meses. Parosmias são comuns. Amnésia nas primeiras 24 horas está associada à anosmia permanente em mais de $90 \%$ dos casos. Quando há preservação parcial da olfação tem-se observado diminuição da discriminação dos odores (17).

A causa exata ainda não foi estabelecida. A teoria mais popular presume uma lesão dos nervos quando estes deixam o topo da lâmina cribiforme. A lesão pode ser no córtex frontal, pois alguns pacientes além de anosmia pós TCE também apresentam alterações psicossociais. A tomografia computadorizada é geralmente normal, podendo em alguns casos revelar fratura da lâmina cribiforme. A hiposmia ocorre mais em lesão frontal; a anosmia em lesão occipital, cinco vezes mais frequente. Cerca de 8 a $39 \%$ dos pacientes recuperam a função, dos quais $75 \%$ nos três primeiros meses (17).

O limiar olfatório diminui com a idade ( $1 \%$ ao ano), sendo esse efeito menor nas mulheres que nos homens. Os idosos têm uma taxa maior de declínio da olfação para uns odores do que para outros, com diminuição da habilidade para discriminar o sabor na comida do cotidiano. Esta diminuição olfatória se deve ao processo fisiológico de envelhecimento (presbiosmia), ocorrendo na sexta ou sétima década, ou às doenças de Alzheimer e Parkinson (18).

A disfunção olfatória éum dos sinais mais prevalentes na Doença de Parkinson. Observam-se alterações de discriminação, identificação e limiar olfatório. A hiposmia é um dos sinais que pode anteceder os sintomas motores da patologia. Em uma pesquisa recente foi encontrado que $80 \%$ dos pacientes com esta patologia apresentaram anormalidade da identificação olfatória, comparados aos controles (20-23).

Na anosmia congênita, a possível fisiopatologia seria a degeneração ou atrofia do epitélio e/ou bulbo olfatório no processo de desenvolvimento. Geralmente é um achado isolado, mas há anosmia familiar associada a calvície prematura e cefaleia vascular, sendo hereditária, dominante, com penetrância variável. A Síndrome de Kallmann é a causa mais comum de disfunção olfatória congênita, 1/1000050000), com anosmia (agenesia do bulbo olfatório) e hipogonadismo hipogonadotrófico, além de a normalidades renais, criptorquidismo, surdez, deformidades médio- 
faciais e diabetes. É causada por um defeito na migração dos neurônios que produzem o hormônio de liberação de gonadotrofinas (GnRH) e dos neurônios que formam os nervos olfatórios. A anosmia está relacionada à deficiência de GnRH porque a migração e diferenciação dos neurônios secretores de GnRH dependem da formação do bulbo olfatório. Os indivíduos acometidos não entendem o conceito de odor, portanto não sentem a sua falta. Pelo fato de geralmente ainda persistirem alguns quimiorreceptores intactos, odores acres, irritantes e gustação podem ser detectados normalmente $(24,25)$.

Quando há exposição do sistema olfatório a substâncias tóxicas, a perda olfatória pode ocorrer em dias ou anos, podendo ser reversível ou permanente. O grau de lesão parece estar relacionado ao tempo de exposição e à concentração e toxicidade do agente, comumente associado ao tabaco. São exemplos de drogas que afetam a olfação: anfetaminas, antibióticos (aminoglicosídeos, tetraciclina), cocaína, derivados de petróleo, dióxido sulfúrico, etanol, formaldeido, metais pesados, metanol, monóxido de carbono, nicotina, solventes orgânicos, sulfato de zinco (tópico) e tetracloreto de carbono $(12,13)$.

Os medicamentos costumam afetar mais a gustação que a olfação. Na maior parte das vezes a olfação retorna com a suspensão da medicação, mas existem relatos de lesão permanente. Drogas que afetam a composição do muco podem alterar a olfação, como os beta-adrenérgicos, colinérgicos e agentes peptidérgicos $(12,13)$.

Os processos neoplásicos também merecem atenção, destacando-se os de localização intranasal, como pólipos nasais, papiloma, carcinoma epidermoide, adenoma, estesioneuroblastoma (tumor neuroolfativo raro), pois bloqueiam o fluxo aéreo para fenda olfatória ou por destruição local do aparelho olfatório (26).

As neoplasias intracranianas que envolvem a superfície orbital do cérebro podem causar anosmia unilateral. Meningiomas da crista esfenoidal ou do sulco olfatório e gliomas do lobo frontal podem lesar os bulbos ou os tratos olfatórios. Anosmia pode também ocorrer em associação a outros tumores do lobo frontal e a lesões parasselares e hipofisárias. Em meningiomas do sulco olfatório ou da área da lâmina cribiforme, anosmia unilateral ocorre precocemente, evoluindo para anosmia bilateral, acompanhada com frequência de neuropatia óptica. A síndrome de Foster Kennedy consiste em anosmia acompanhada de atrofia óptica ipsilateral unilateral e papiledema contralateral, oriunda classicamente de um grande tumor envolvendo a região orbitofrontal (26).

Certas patologias psiquiátricas cursam com distúrbios da olfação. A esquizofrenia pode cursar com alucinações olfatórias em $15 \%$ a 30\% das vezes. Pacientes com depressão maior podem apresentar mesmo sintoma, mas geralmente possui habilidade olfatória preservada. A fantosmia pode se apresentar como aura em pacientes com epilepsia do lobo temporal $(15,18)$.

A iatrogenia não pode deixar de ser mencionada como fator etiológico relevante. Em procedimentos cirúrgicos pode ocorrer dano neural e estreitamento do fluxo nasal por alterações anatômicas ou tecido cicatricial. Alterações no olfato e no paladar ocorrem após laringectomia total, pois o paciente passa a respirar diretamente pela traqueia e o ar não passa através do nariz para os órgãos olfativos terminais. Como o olfato e o paladar estão intimamente ligados, as sensações de paladar são alteradas. Mas, com o passar do tempo, o paciente comumente se acomoda a este problema, o que pode justificar o fato de nem todos os pacientes referirem alteração olfatória (2729).

Em cirurgias da fossa anterior e pós neurocirurgia transesfenoidal pode ocorrer lesão de lâmina cribiforme. A radioterapia também está inclusa no conjunto de condições que levam a disfunções do olfato e paladar, assim como as de causas idiopáticas, geralmente em adultos jovens, na meia idade e saudáveis $(12,26)$.

Na Hanseníase, as alterações de olfato podem ser encontradas em qualquer forma clínica da doença. Além disso, é uma queixa muito comum nessa patologia e pode ser encontrada mais frequentemente na forma lepromatosa, sendo referido que este acometimento estivesse relacionado com a severidade das alterações clínicas na mucosa nasal. Em estudo realizado em 2005, os achados de alterações de olfato foram encontrados em quatro formas diferentes de hanseníase, porém em pacientes em estágio avançado ou em reação. Encontrou-se hiposmia em 7,5\% dos pacientes, cacosmia em 2,3\% e anosmia em 0,6\% (16, 30, 31).

Poucos casos de distúrbio do olfato têm origem neurológica. Esclerose múltipla pode causar alterações do olfato devido a envolvimento das vias olfatórias. Condições neurológicas diversas que causam anosmia incluem hidrocefalia, acometimento da artéria cerebral anterior próximo à sua origem, meningite basilar, abscessos do lobo frontal e doença de Refsum. Lobectomias temporais que incluam o córtex piriforme podem causar déficits na identificação de odores (26).

A hiperosmia geralmente é funcional, mas pode ocorrer em certos tipos de abuso de drogas e enxaqueca. Alucinações olfativas se devem mais frequentemente a psicose, mas podem decorrer de uma lesão do sistema olfativo central, geralmente neoplásica ou vascular, ou 
como manifestação de crise convulsiva. As assim chamadas crises uncinadas são crises parciais complexas ou do lobo temporal precedidas de uma aura olfativa ou gustativa, geralmente desagradável, e frequentemente acompanhadas, enquanto o paciente perde a consciência, de movimentos de estalar os lábios e ou de mastigação. Esses ataques são tipicamente oriundos de um foco convulsivo envolvendo estruturas do lobo temporal medial (26).

O paladar pode ser afetado em casos de lesões do nervo facial proximais à saída da corda timpânica. Já no caso de distúrbios gustativos permanentes, estes podem sobrevir após paralisia facial de Bell. Disfunções do paladar e do olfato frequentemente ocorrem juntas, pois as anormalidades do paladar se devem geralmente a disfunção olfativa. Disgeusia pode ser um efeito direto ou indireto de condições malignas. Hipergeusia e parageusias podem ocorrer em psicoses e no transtorno de conversão (32).

Alucinações gustativas podem ocorrer em crises parciais complexas e nos tumores envolvendo o uncus ou o opérculo parietal e frequentemente ocorrem em conjunto com as alucinações olfatórias. Pacientes idosos desenvolvem por vezes disgeusia de origem obscura que pode ocasionar anorexia e perda de peso. A sensibilidade gustativa aumentada ocorre em pacientes com doença de Addison, deficiência da hipófise e fibrose cística. Lesões do nervo lingual podem causar perda do paladar juntamente com perda da sensação exteroceptiva do lado da língua afetado (32).

\section{Métodos Diagnósticos}

A avaliação das alterações do olfato pode ser feita através da apresentação de odores (canela, aguarrás, limão, fumaça, chocolate, rosa, solvente de tinta, banana, abacaxi, gasolina, sabonete, cebola). Cada narina deve ser explorada separadamente, e o paciente é interrogado sobre o tipo de odor $(21,33)$.

Uma anamnese detalhada deve ser realizada, esclarecendo sobre doenças na família, cirurgias anteriores, traumas cranianos, exposição e/ou uso de drogas. Microscopia ou endoscopia pode definir alterações do fluxo aéreo como causa de anosmia (33).

A endoscopia nasal éútil no acesso à fenda olfatória, sendo em conjunto com a tomografia computadorizada os meios mais sensíveis para o diagnóstico de patologias derivadas da cavidade nasal, seios paranasais e encéfalo. A rinometria apresenta pouco valor diagnóstico, servindo apenas para ilustrar diminuições do fluxo respiratório. A ressonância nuclear magnética é útil para avaliação do bulbo olfatório, tratos olfatórios e causas intracranianas de distúrbios da olfação (34).
Tsukatani et al em 2005 demonstrou que diferentes testes são concordantes em avaliar se há ou não disfunção no olfato, mas os testes não são concordantes em avaliar os níveis de hiposmia (34).

Nos testes de detecção busca-se a menor concentração do odorífero capaz de ser detectado. São realizados oferecendo ao paciente dois ou mais estímulos, sendo que apenas um possui substância com odor. Esse tipo de investigação mostrou-se mais efetiva que simplesmente perguntar se um odor pode ou não ser sentido (35).

Nos testes de reconhecimento busca-se a menor concentração do odor capaz de ser reconhecido, sendo mais utilizado o método ascendente de limiar. Nesse teste, os odores são apresentados sequencialmente da menor para a maior concentração e assim estima-se o ponto de reconhecimento do odor (35).

Ressalta-se que tanto o teste de detecção como o de reconhecimento são métodos subjetivos e dependem de fatores como idade, cooperação do examinado e graus de compreensão do mesmo, com duração média de 20 a 30 minutos para sua correta execução (35).

BRINER \& SIMMEN em 1999 descreveram um teste de screening da olfação utilizando oito disquetes contendo diferentes odores (5), que são abertos para liberar o odor e fechados após o teste, sendo atribuído um (01) ponto para cada acerto do paciente. Considera-se como normal os valores de 6,2 $\pm 1,0$ para o grupo etário entre 18 e 50 anos e de 6,0 \pm 0,9 para o grupo entre 51 e 80 anos. É um método simples, de rápida execução e elimina o risco de contaminação das mãos do examinador e do paciente pelo odor. No entanto, ocorre uma intensa liberação do odor ao se abrir o disquete, sendo portanto um teste supralimiar, servindo-se como screnning (36).

O "University of Pennsylvania Smell Identification Test" (UPSIT) é um teste psicofísico (subjetivo), com ampla utilização. São oferecidos 50 odores diferentes ao paciente através de uma cartela que, ao ser riscada, exala um odor. A seguir são analisadas: intensidade, irritação, frio, familiaridade e agradabilidade (37).

O "Modular Smell Identification Test" (MODSIT) é uma variante do método UPSIT, porém com menor custo e tempo de realização do exame, pois são oferecidos 12 odores para o paciente que necessita ser alfabetizado para leitura da cartela (38).

São descritos na literatura aparelhos para a medida subjetiva da olfação como o T\&T olfatômetro, que consiste de pequenos frascos contendo diluições de 5 diferentes odores, sendo utilizado para determinar o limiar de detecção 
e o reconhecimento de cada estímulo, obtendo-se um valor médio do limiar e o olfatômetro Jet Stream (corrente em jato), que se constitui em três partes: um adaptador para a fossa nasal, um dispositivo para a colocação e diluição do odorífero num tubo e um mini compressor de ar $(39,40)$.

Outros exames podem auxiliar no diagnostico de disfunção olfatoria tais como: Tomografia Computadorizada com Emissão Única de Fótons (SPECT), Reflexo olfatóriopupilar, Reflexo olfatório-tensional ou cardiovascular, Reflexo cutâneo ou psicogalvânico, Reflexo olfatório-respiratório, eletrolfatograma e o Potencial evocado do nervo olfatório (4).

Anamnese detalhada também é importante para o diagnóstico das alterações do sabor. Em algumas situações, é necessário o auxílio de outros especialistas, como endocrinologista e geneticista, para melhor esclarecimento diagnóstico. Papéis embebidos em soluções com diferentes concentrações de glicose, sal, ácido, etc, podem ser utilizados, ou pode-se empregar a eletrogustometria, que consiste na estimulação da língua com correntes elétricas, causando ao paciente a sensação de gosto ácido e/ou metálico (33).

\section{Tratamento}

Muitas ideias simples podem ser sugeridas a pacientes com alterações de gosto. A mastigação de goma ou de gelo pode atuar como uma ajuda temporária na hipogeusia. Os pacientes devem ser encorajados a mastigar seus alimentos muito bem, alterando os lados de sua boca ou então os seus alimentos (41).

Os distúrbios da quimiossensibilidade, olfação e gustação, são sintomas de doenças, por isso o tratamento depende da sua causa. As alterações do olfato causadas por infecções virais são tratadas com hidratação oral, repouso e analgésico, se necessário. Nas causas obstrutivas nasais, a correção cirúrgica conjuntamente com o emprego de corticosteroides tópicos tem se mostrado eficaz. Nas perdas por trauma, as alterações gustativas e olfativas, se não regredirem após a melhora do edema, geralmente são irreversíveis (33).

Em muitos casos o tratamento das alterações olfatórias e gustativas não é fácil e é necessária uma cooperação interdisciplinar entre o otorrinolaringologista, endocrinologista, neurologista, psiquiatra entre outros (41).

\section{COMENTÁRIOS FinAIS}

A olfação e a gustação apresentam anatomia e fisiologia complexas, ainda não totalmente conhecidas. Sua importância para os animais e seres humanos é vital e sua perda traz graves consequências na qualidade de vida, bem como pode representar risco à saúde do indivíduo. Um dano ao sistema olfatório pode atenuar a percepção do sabor já que estes sistemas apresentam uma íntima ligação. É essencial o conhecimento dos testes atualmente disponíveis para que se possa realizar um diagnóstico preciso e responder às dúvidas do paciente com relação à perda parcial ou total do olfato e gustação. Deve-se ter em mente que a detecção precoce dessas alterações pode levar a um tratamento mais efetivo, no intuito de retardar a progressão das doenças que ocasionam perda olfatória e gustatória e desta forma atenuar a severidade dos sintomas.

\section{REFERÊNCIAS BIBLIOGRÁFICAS}

1. Tortora GJ, Grabowski SR. Corpo Humano: Fundamentos de Anatomia e Fisiologia. 6⿳a ed. Porto Alegre (RS): Artmed; 2005.

2. Pellegrini G, Veleiro RVB, Gomes ICD. A percepção do gosto salgado em indivíduos com e sem obstrução nasal. Rev. CEFAC 2005, 7(3):311-7.

3. Hungria, H. Otorrinolaringologia. $8^{\mathrm{a}}$ ed. Rio de Janeiro (RJ): Guanabara Koogan; 2000.

4. Rocha FMN, Ximenes Filho JA, Alvarenga EHL, Mello Jr JF. Olfação: revisão de literatura. Arq Int Otorrinolaringol. 2002, 6(2):123-8.

5. D'Ottaviano EJ. Sistema nervoso e $3^{\text {a }}$ idade: $2^{\underline{a}}$ parte. Rev. das Faculdades de Educação, Ciências e Letras e Psicologia Padre Anchieta. 2001, 3(5):19.

6. Almeida MM, Freire GL, Morais LCSL. Implantação e avaliação da prática: "cansando o olfato". In: Encontro de iniciação científica à docência, 11; 2008 outubro; Paraíba. Anais. p. 33 [resumo 002].

7. Weckx LLM. Consenso sobre rinites. Rev Bras de Otorrinolaringol. 2000, 66(3):1-34.

8. Toneloto C. Mal de Parkinson: teste do olfato auxilia no diagnóstico precoce da síndrome. Ciencia e Cultura. 2007, 59(3):11-3.

9. Buck LB. Olfação e gustação: os sentidos químicos. In: Kandel ER, Schwartz JH. Princípios da neurociência. $4^{\mathrm{a}} \mathrm{ed}$. Barueri: Manole; 2002. p.625-47.

10. Guyton AC, Hall,JE. Os sentidos químicos: gustação e olfação. In: Guyton AC, Hall, JE. Tratado de fisiologia médica. 10ª ed. Rio de Janeiro: Guanabara Koogam SA; 2002. p.570-7. 
11. Junqueira LC, Carneiro J. Histologia básica. 10ํe ed. Rio de Janeiro (RJ): Guanabara Koogam S.A.; 2004.

12. Andreas FP, Temmel MD, Christian QMD, Bettina SFMD, Ludger KMD, Hummel EST. Characteristics of olfactory disorders in relation to major causes of olfactory loss. Arch Otolaryngol Head Neck Surg. 2002, 128(8):635-41.

13. Daniel A, Kimmelman CP, Mester AF, Brightman VJ, Settle GR, Snow JB et al. Smell and taste disorders, a study of 750 patients from the University of Pennsylvania. Otolaryngol Head Neck Surg. 1991, 117(5):519-28.

14. Hummel T, Nordin S. Olfactory disorders and their consequences for quality of life. Acta Oto-Laryngologica. 2005, 125(2):116-21.

15. Kopala LC, Good KP. Olfactory deficits in patients with schizofrenia and severe polydipsia. Biol Psychiatry. 1998, 43(7):497-502.

16. Kern RC. Chronic sinusitis and anosmia: pathologic changes in the olfactory mucosa. Laryngoscope. 2000, 110(7):1071-7.

17. Mueller A, Rodewald A, Reden J, Gerber J, von Kummer $\mathrm{R}$, Hummel T. Reduced olfactory bulb volume in posttraumatic and post-infectious olfactory dysfunction. Neuroreport. 2005, 16(5):475-8.

18. Solomon GS, Petrie WM, Hart JR, Brackin HB. Olfatory dysfunction Discriminates Alzheimers dementia from major depression. J Neuropsychiatry Clin Neurosci. 1998, 10(1): 64-7.

19. Weckx UM, Sakano E, Araújo E, Castro F, Aun W. Consenso sobre Rinites. Rev Bras Otorrinolaringol. 2000, 66 (supl. 10):1-34.

20. Katzenschlager R, Lees AJ. Olfaction and Parkinson's syndromes: its role in differential diagnosis. Curr Opin Neurol. 2004, 17(4):417-23.

21. Quagliato LB, Viana MA, Quagliato EMAB, Simis S. Alterações do olfato na doença de Parkinson. Arq Neuropsiquiatr. 2007, 65(3):647-52.

22. Doty RJ, Bromley SM, Stern MB. Olfactory testing as an aid in the diagnosis of Parkinson's disease: development of optimal discrimination criteria. Neurodegeneration. 1995, 4:93-7.

23. Katzenschlager R, Lees AJ. Olfaction and Parkinson's syndromes: its role in differential diagnosis. Curr Opin Neurol. 2004, 17:417-23.
24. Ribeiro RS, Abucham J. Síndrome de Kallmann: uma revisão histórica, clínica e molecular. Arq Bras Endocrinol Metab. 2008, 52(1):8-17.

25. Schmidt VB, Roithmann R, Corleta HE, Capp E. Hipogonadismo hipogonadotrófico e anosmia: síndrome de Kallmann. Rev Bras Otorrinolaringol. 2001, 67(6):880-4.

26. Campbell, WW. O nervo olfativo. In: Campbell, WW. DeJong, o exame neurológico. $6^{\underline{a}}$ ed. Rio de Janeiro: Guanabara Koogan; 2007. p.97.

27. Smeltzer SC, Bare BG. Brunner \& Suddarth: tratado de enfermagem médico-cirúrgica. $8^{a}$ ed. Rio de Janeiro (RJ): Guanabara Koogan S.A; 1998.

28. Silva LSL, Pinto MH, Zago MMF. Assistência de enfermagem ao laringectomizado no período pósoperatório. Rev Bras de Cancerol. 2002, 48(2):213-21.

29. Ghirardi, ACAM. Laringectomizados usuários de prótese traqueoesofágica: princípios e métodos da prática fonoaudiológica [Tese-Mestrado]. São Paulo (SP): Pontifícia Universidade Católica de São Paulo; 2007.

30. Martins ACC, Castro JC, Moreira JS. Estudo retrospectivo de dez anos em endoscopia das cavidades nasais de pacientes com hanseníase. Rev Bras Otorrinolaringol. 2005, 71(5):609-16.

31. Barton RPE, Davey TF. Early leprosy of the nose and throat. J Laryngol Otol. 1976, 90(10):953-61.

32. Campbell, WW. O nervo facial. In: Campbell, WW. DeJong, o exame neurológico. $6^{a}$ ed. Rio de Janeiro: Guanabara Koogan; 2007. p.190.

33. Maffeis ER, Netto SCR. Fatores que alteram a percepção gustativa. Rev Fac Odontol Lins. 1990, 3(2):28-32.

34. Tsukatani T, Reiter, ER, Miwa T, Costanzo RM. Comparison of Diagnostic Findings using Different Olfactory Test Methods. Laryngoscope. 2005, 115(6):1114-1117.

35. Doty RL, Kobal G. Current trends in the measurement of olfactory function. Handbook of olfaction and gestation. 1995; 8: 191-225.

36. Briner HR, Simmen D. Smell diskettes as screening test of olfaction. Rhinology. 1999, 37:145-148.

37. Doty RL, Shaman P, Dann M. Development of the University of Pennsylvania Smell Identification Test: A Standardized Microencapsulated Test of Olfactory Function. Physiology \& Behavior. 1984, 32:489-502. 
38. Liu HC, Wang SJ, Lin KP, Lin KN, Fuh JL, Teng EL. Performance on a smell screening test (the MODSIT): A study of 510 predominantly illiterate Chinese subjects. Physiology \& Behavior. 1995, 58(6):1251-5.

39. Kondo H, Matsuda T, Hashiba M, Baba S. A study of the relationship between the T\&T Olfactometer and the University of Pennsylvania Smell Identification Test in a Japanese population. Am J Rhinology. 1998, 12(5):353-8.
40. Ikeda K, Tabata K, Oshim T, Nishikawa H, Hidaka H, Takasaka T. Unilateral examination of olfactory threshold using the Jet Stream Olfactometer. Auris Nasus Larynx. 1999, 26:435-9.

41. Burkert S, Haberland EJ, Gudziol H. Olfactory and gustatory disorders: causes, diagnosis and treatment. MMW Fortschr Med. 2005, 147(11):51-3 